\title{
Mathematical Model and Simulations of COVID-19 2020 Outbreak in New York: Predictions and Implications for Control Measures
}

\section{Durgesh Nandini Sinha ${ }^{1 *}$ and Peiwen Tan ${ }^{2}$}

${ }^{1}$ Adjunct Assistant Professor, Temple University, Philadelphia/Strayer University (Online)/Rowan College at Burlington County, New Jersey/Community College of Philadelphia/Mercer County Community College, New Jersey, USA ${ }^{2}$ Lecturer , University of Pennsylvania, Philadelphia, PA / Adjunct Assistant Professor, Temple University, Philadelphia, PA, USA

*Corresponding Author: Durgesh N Sinha, Adjunct Assistant Professor, Temple University, Philadelphia/Strayer University (Online)/Rowan College at Burlington County, New Jersey/Community College of Philadelphia/Mercer County Community College, New Jersey, USA.

DOI: $10.31080 /$ ASMI.2020.03.0646
Received: May 20, 2020

Published: July 10, 2020

(C) All rights are reserved by Durgesh

Nandini Sinha and Peiwen Tan.

\section{Abstract}

The outbreak of the novel coronavirus has resulted in significant morbidity and mortality in the affected 210 countries with about 2.4 million people infected and over 163 thousand deaths. The SARS-CoV-2 spike protein is effective at binding to human cells, but this SARS-CoV-2 backbone differed substantially from those of already known coronaviruses and mostly resembled related viruses found in bats and pangolins. To help predict the possible dynamics of COVID-19 as well as ways to contain it, this paper develops a mathematical model for the disease, which includes two different infectious routes. The model's predictions are fitted to data from the outbreaks in New York State from March 12020 first report to April 19 2020. However, the containment time and the severity of the outbreaks depend crucially on the contact coefficients and the isolation rate constant. When randomness is added to the model coefficients, the simulations show that the model is sensitive to the scaled contact rate among people and to the isolation rate. The model is analyzed using stability theory for ordinary differential equations and indicates that when using only isolation for control and advising self recovery,the endemic steady state is locally stable and attractive. After the April 142020 highest peak of COVID-19 new infections by the SARS-CoV-2 virus will slow down from the beginning of May at New York State if people will keep the isolation. Numerical simulations with parameters estimated from New York State illustrate the analytical results and the model behavior, which may have important implications for the disease containment in other cities. Indeed, the model highlights the importance of isolation of infected individuals and advising self recovery may be used to assess other control measures. The model is general and may be used to analyze outbreaks in other states of the United States and other countries.

Keywords: COVID-19; SARS-CoV-2; NY Population; SEIQRD Model; Peak Prediction; Dynamical System; Reproduction Number

\section{Introduction}

The epidemic of 2019 novel coronavirus (now called SARSCoV-2, causing the disease COVID-19) has expanded from Wuhan to the entire country of China and then has subsequently been spread to other countries that now have exponential growth in cases; thousands of thousands of people have seen onward transmis- sion. Early efforts have focused on describing the clinical course, counting severe cases, and treating the sick. Experience with the Middle East respiratory syndrome (MERS), pandemic influenza, NIPAH (similar host bats) [4] and other outbreaks has shown that as an epidemic evolves, we face an urgent need to expand public health activities in order to elucidate the epidemiology of the novel 
virus and characterize its potential impact. The impact of an epidemic depends on the number of persons infected, the infection's transmissibility, and the spectrum of clinical severity.

New York's coronavirus outbreak has violently erupted over the past few days, and the state is now driving the national epidemic while on the West Coast, public health experts are wondering if an early and aggressive response saved California from a similar fate.

Thousands of new COVID-19 infections are now being reported in New York every day. As of Sunday night (04/18/2020), there have been at least 242,786 confirmed cases of the coronavirus discovered in the state. At least 13,865 people with COVID-19 have died in the state, which has the largest number around 30 percent of total confirmed cases in the U.S today; before the high peak the affected number was $45 \%$ of the US.

Yet, are we entitled to use the whole of our minds, and therefore the technological knowhow and the economic resources to tamper with an epidemiological climax?

These approaches appear reasonable, but they have prompted in recent years cyclical phases of panic and of unjustified fear, that strongly impacted the economy and the social systems. This led to a reactive rather than a proactive strategy piloting the resources towards the research of tailored measures aimed to produce acute responses to the crises. Nevertheless, this approach resulted, in the best case, in the production of vaccines and antiviral drugs whose use - although extremely welcome - may be effective only when an epidemic is already in place.

\section{Evidence for natural evolution}

The scientists found that the RBD portion of the SARS-CoV-2 spike proteins had evolved to effectively target a molecular feature on the outside of human cells called ACE2, a receptor involved in regulating blood pressure. The SARS-CoV-2 spike protein was so effective at binding the human cells, in fact, that the scientists concluded it was the result of natural selection and not the product of genetic engineering. (Genetic Engineering and Biotechnology News).

This evidence for natural evolution was supported by data on SARS-CoV-2's backbone -- its overall molecular structure. If some- one were seeking to engineer a new coronavirus as a pathogen, they would have constructed it from the backbone of a virus known to cause illness. But the scientists found that the SARS-CoV-2 backbone differed substantially from those of already known coronaviruses and mostly resembled related viruses found in bats and pangolins.

"These two features of the virus, the mutations in the RBD portion of the spike protein and its distinct backbone, rule out laboratory manipulation as a potential origin for SARS-CoV-2" said Andersen.

Josie Golding, PhD, epidemics lead at UK-based Welcome Trust, said the findings by Andersen and his colleagues are "crucially important to bring an evidence-based view to the rumors that have been circulating about the origins of the virus (SARS-CoV-2) causing COVID-19".

"They conclude that the virus is the product of natural evolution," Goulding adds, "ending any speculation about deliberate genetic engineering".

\section{Possible origins of the virus}

Based on their genomic sequencing analysis, Andersen and his collaborators concluded that the most likely origins for SARS-CoV-2 followed one of two possible scenarios.

In one scenario, the virus evolved to its current pathogenic state through natural selection in a non-human host and then jumped to humans. This is how previous coronavirus outbreaks have emerged, with humans contracting the virus after direct exposure to civets (SARS) and camels (MERS). The researchers proposed bats as the most likely reservoir for SARS-CoV-2 as it is very similar to a bat coronavirus. There are no documented cases of direct bat-human transmission, however, suggesting that an intermediate host was likely involved between bats and humans.

In this scenario, both of the distinctive features of SARS-CoV-2's spike protein -- the RBD portion that binds to cells and the cleavage site that opens the virus up -- would have evolved to their current state prior to entering humans. In this case, the current epidemic would probably have emerged rapidly as soon as humans were infected, as the virus would have already evolved the features that make it pathogenic and able to spread between people. 
In the other proposed scenario, a non-pathogenic version of the virus jumped from an animal host into humans and then evolved to its current pathogenic state within the human population. For instance, some coronaviruses from pangolins, armadillo-like mammals found in Asia and Africa, have an RBD structure very similar to that of SARS-CoV-2. A coronavirus from a pangolin could possibly have been transmitted to a human, either directly or through an intermediary host such as civets or ferrets.

Then the other distinct spike protein characteristic of SARSCoV-2, the cleavage site, could have evolved within a human host, possibly via limited undetected circulation in the human population prior to the beginning of the epidemic. The researchers found that the SARS-CoV-2 cleavage site, appears similar to the cleavage sites of strains of bird flu that has been shown to transmit easily between people. SARS-CoV-2 could have evolved such a virulent cleavage site in human cells and soon kicked off the current epidemic, as the coronavirus would possibly have become far more capable of spreading between people.

Study co-author Andrew Rambaut cautioned that it is difficult if not impossible to know at this point which of the scenarios is most likely. If the SARS-CoV-2 entered humans in its current pathogenic form from an animal source, it raises the probability of future outbreaks, as the illness-causing strain of the virus could still be circulating in the animal population and might once again jump into humans. The chances are lower of a non-pathogenic coronavirus entering the human population and then evolving properties similar to SARS-CoV-2.

The purpose of our work is to develop a mathematical model [4] of how the virus evolved to its current pathogenic state through natural selection in a non-human host and then jumped to humans or possibly via limited undetected circulation in the human population prior to the beginning of the epidemic.

SARS-CoV-2 can be transmitted to humans via both animals and humans, and it spreads among people who are in close contact. Transmission from infected patients to healthcare personnel has also been observed. Confirmed cases include only those with a positive polymerase chain reaction in accordance with the laboratory guidelines for virus genetic material. Probable cases are those that have a link with a confirmed case and a clinically compatible illness but without definitive laboratory confirmation. The major- ity of patients experienced severe respiratory disease while the minority were reported to have non-severe disease and some cases were reported as asymptomatic. Severe disease was defined as admission to an intensive care unit, use of extracorporeal membrane oxygenation, mechanical ventilation and vasopressors. The signs and symptoms include fever, cough, sore throat, chills, and shortness of breath. Some patients progress to dyspnea and pneumonia. Complications of the disease include severe pneumonia with acute respiratory distress syndrome, septic shock, and multi-organ failure that result in death. This work develops a compartmental mathematical model for COVID-19, which is based on models for SARS [1] and MERS [2,3].

Serious illness, death increase with advancing age.

The first description of outcomes in 4,226 US COVID-19 cases reported to the CDC from Feb 12 to Mar 16 shows that $31 \%$ of cases, $45 \%$ of hospitalizations, $53 \%$ of intensive care unit (ICU) admissions, and $80 \%$ of deaths occurred in people 65 years or older.

Fatality rates for people 85 years and older ranged from $10 \%$ to $27 \%$. Of those aged 65 to 84 years, $3 \%$ to $11 \%$ died. Death rates fell to $1 \%$ to $3 \%$ among those 55 to 64 , less than $1 \%$ in those 20 to 54 , and $0 \%$ in those 19 and younger.

\section{COVID-19 persists in the air, on surfaces}

The virus that causes coronavirus disease 2019 (COVID-19) is stable for several hours to days in aerosols and on surfaces, according to a new study from National Institutes of Health, CDC, UCLA and Princeton University scientists in The New England Journal of Medicine. The scientists found that severe acute respiratory syndrome coronavirus 2 (SARS-CoV-2) was detectable in aerosols for up to three hours, up to four hours on copper, up to 24 hours on cardboard and up to two to three days on plastic and stainless steel. The results provide key information about the stability of SARSCoV-2, which causes COVID-19 disease, and suggests that people may acquire the virus through the air and after touching contaminated objects.

To study the dynamics of the disease and to assess its spread and the effectiveness of various measures to contain or eradicate it, this work develops a compartmental mathematical model for SARSCoV-2, which is a developed model from SARS [1] and MERS [2,3]. 
We choose to use a continuous model and not a stochastic one, although the data was discontinuous and random, since our model captures the dynamics of the diseases well and avoids some of the mathematical difficulties associated with stochastic differential equations. The model describes the dynamics of five populations consisting of susceptible, asymptomatic or exposed individuals (individuals who carry the virus and can infect others but have no symptoms), infectious individuals with symptoms, isolated and recovered individuals. The virus dynamics and interactions with animals that may carry it are not included in the model at this initial stage of its development.

Mathematically, the existence of a unique solution for the system is straightforward, and here the non- negativity of the solution is shown. It is found that the model has a disease-free equilibrium and may have an endemic equilibrium.

\section{Methods}

Data sources

To estimate the early dynamics of transmission in New York, we fitted a stochastic transmission dynamic model to observe data from March 1, 2020 to April 18, 2020 by the total number of confirmed cases, new cases reported each day, total number of deaths, and new deaths reported each day. Data has been observed from the source $[9,10]$.

\section{Procedures}

In the model, we divided individuals into six classes, as follows: susceptible, exposed (but not yet infectious), infectious (those confirmed positive after test), quarantine (infectious plus all infectious contacted ), recovered (no longer infectious), and deceased (those died in infectious class or quarantine class). We assumed here those confirmed infectious will recover from two routes, one from taking treatments at home while isolated, and others whose symptoms required hospitalization. The incubation period was used from 2 to 14 days. The delay in hospitalization from confirmed cases depending on severity is used one day. Death rate is considered one who died at hospital, and others who died in home (or an isolation place). The death in hospitalization is $2.5 \%$ of confirmed cases, while at home is $0.915 \%$ with $95 \%$ CI $0.91 \%$, 0.92\%) (LHS PRCC fitted) of confirmed cases.
Model formation and parameter classifications

We divided the human population into four classes, SEIQRD (Susceptible-Exposed-Infected-Quarantine-Recovered-Deceased).

Schematic flow of this model is shown in figure 2 and the state variables and associated parameters of this model are given in table 1.

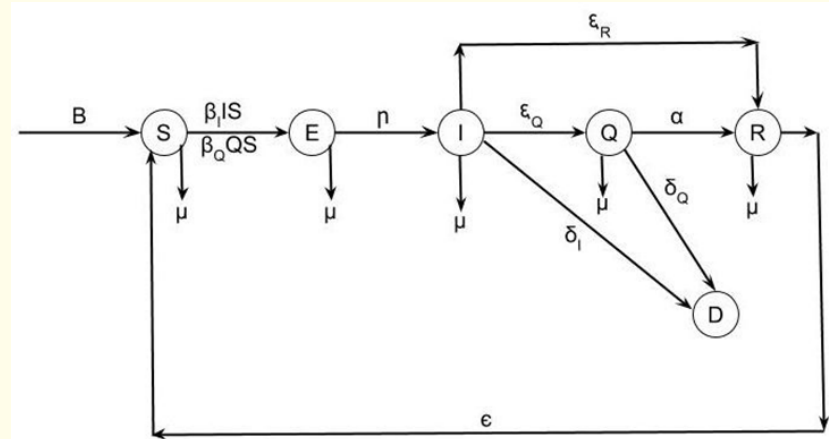

Figure 1

$\mathrm{S}(\mathrm{t})$ : Susceptible humans in time $\mathrm{t}$

$\mathrm{E}(\mathrm{t})$ : Exposed humans in time $\mathrm{t}$

$\mathrm{I}(\mathrm{t})$ : Infectious humans in time $\mathrm{t}$

$\mathrm{Q}(\mathrm{t})$ : Quarantine human in time

$R(t)$ : Recovered humans with Immunity in time $t$

$D(t)$ : Cumulative Deceased humans in time $t$

$\mathrm{N}(\mathrm{t})$ : Total human population in time $\mathrm{t}$

B: Birth rate of humans

$\beta$ i: Infectivity rate of COVID-19 from Symptotic infectious class

$\beta Q$ : Infectivity rate of COVID-19 from quarantine population class $\eta$ : Rate of transition from exposed to infected humans

$\alpha$ : Rate of transition from Quarantine to recovered humans

$\varepsilon$ : Rate of reinfection from Immuned (Recovered) humans from COVID-19

छr: Rate of transition from Infected class to Recovered class

$\xi_{0}$ : Rate of transition from infected class to Quarantine infected class

$\mu$ : Natural death rate of humans

$\delta$ i: Death rate of humans due to COVID-19 from Infected class

$\delta_{\mathrm{o}}$ : Death rate of human due to COVID-19 from Quarantined class. 


$$
\begin{aligned}
& \frac{\mathrm{d} S}{\mathrm{~d} t}=B N-\beta_{i} I S-\beta_{Q} Q S-\mu S+\varepsilon R \\
& \frac{\mathrm{d} E}{\mathrm{~d} t}=\beta_{i} I S+\beta_{Q} Q S-(\eta+\mu) E \\
& \frac{\mathrm{d} I}{\mathrm{~d} t}=\eta E-\left(\xi_{Q}+\xi_{R}+\delta_{i}+\mu\right) I \\
& \frac{\mathrm{d} Q}{\mathrm{~d} t}=\xi_{Q} I-\left(\mu+\delta_{Q}+\alpha\right) Q \\
& \frac{\mathrm{d} R}{\mathrm{~d} t}=\xi_{R} I-\alpha Q-(\mu+\varepsilon) R \\
& \frac{\mathrm{d} D}{\mathrm{~d} t}=\delta_{i} I+\delta_{Q} Q
\end{aligned}
$$

And

$N(t)=S(t)+E(t)+I(t)+\mathrm{Q}(\mathrm{t})+\mathrm{R}(\mathrm{t})+\mathrm{D}(\mathrm{t})$

All our parameters are positive or non-negative, therefore all parameters must remain positive or non-negative for initial conditions for $\mathrm{t} \geq 0$. From our model we $\mathrm{dN} / \mathrm{dt}=B-\mu N-\delta i I-\delta q Q \leq B$ $-\mu N$. The closed set $\mathrm{D}=\left\{(\mathrm{S}, \mathrm{E}, \mathrm{I}, \mathrm{Q}, \mathrm{R}, \mathrm{D}) \in \mathrm{R}+{ }^{6} ; \mathrm{N} \leq B / \mu\right\}$ is a feasible region for the graph.

Some model parameters were calculated using the Latin Hypercube Sampling partial Correlation Coefficient Method (LHS PRCC). We used this method to calculate all of the infectivity values, $\beta$, as well as the transitions between E, I, Q and D.

We have also proved that our model is locally and globally stable for both diseases-free-equilibrium and endemic equilibrium points through block matrix and Lyapunov function methods that can be provided if needed upon request.

Since all of our model parameters are positive or non-negative, it is important to show that all state variables remain positive or non-negative for all positive initial conditions for $t \geq 0$.

As for the basic reproduction number, it is the average number of secondary infectious cases produced by a single infection in the total susceptible population. The basic reproduction number is calculated by $R_{0}=\mathcal{L}\left(\mathrm{FV}^{-1}\right)$, where $\mathcal{L}$ is the spectral radius of the matrix $\mathrm{FV}^{-1}$, and $\mathrm{F}$ and $\mathrm{V}$ are the matrices of new infection terms and the remaining transmission terms respectively. By solving equation (1) and (2), we get $\mathrm{F}$ and $\mathrm{V}$ of the human population as given below:

$F=\left[\begin{array}{ccc}0 & \beta_{i} & \beta_{Q} \\ 0 & 0 & 0 \\ 0 & 0 & 0\end{array}\right]$
$V=\left[\begin{array}{ccc}(\eta+\mu)) & 0 & 0 \\ -\eta & \left(\xi_{Q}+\xi_{R}+\delta_{i}+\mu\right) & 0 \\ 0 & -\xi_{Q} & \left(\mu+\delta_{Q}+\alpha\right)\end{array}\right]$

Let,

$X_{1}=(\eta+\mu), X_{2}=\left(\xi_{Q}+\xi_{R}+\delta_{i}+\mu\right), X_{3}=\left(\mu+\delta_{Q}+\alpha\right)$

$F V^{-1}=\left[\begin{array}{ccc}\eta \beta_{i} / X_{1} X_{2}+\eta \xi Q \beta_{Q} / X_{1} X_{2} X_{3} & \beta_{i} / X_{2}+\beta_{Q} \xi_{Q} / X_{2} X_{3} & \beta_{Q} / X_{3} \\ 0 & 0 & 0\end{array}\right]$

$R_{0}=\operatorname{Trace}\left(F V^{-1}\right)$

$R_{0}=\frac{\eta \beta_{i}}{X_{1} X_{2}}+\frac{\eta \xi_{Q} \xi_{Q}}{X_{1} X_{2} X_{3}}$

Numerical analysis

Parameter estimation: To evaluate the reproduction number Ro of COVID-19 in the New York population, we applied new infection observed in 2 days to 14 days incubation period interval of data obtained from $[9,10]$ from March 1, 2020 to April 6, 2020. Then on average, $95 \%$ CI calculated of all Ro calculated, and it was found R0 = 27.6156 95\% CI [9.65301, 45.5781] shown in figure 2. Figure 3 depicts the box plots of variation of reproduction number R0 from 2 days incubation to 14 days incubation period. It was found that the data were increasing twice in two days. We estimated that effective reproduction number $\mathrm{R}(\mathrm{t})$ varied during Feb 11, 2020 to April 6, 2020 with median value ranging from $1.45-45$. We estimated a decline in $\mathrm{R}(\mathrm{t}$ ) from late March, from 41.483 (95\% CI 23.07 - 115.32) on March 22, the week the lockdown started, to 1.17 (95\% CI 1.05 - 1.38) on April 6, 2020.

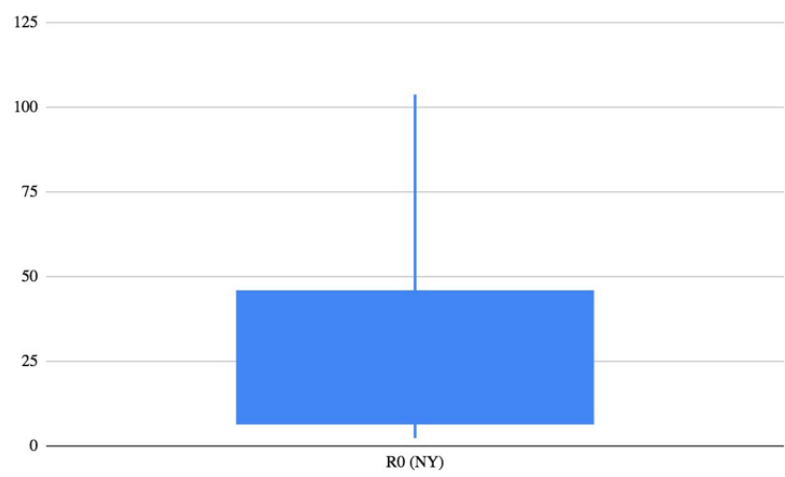

Figure 2 


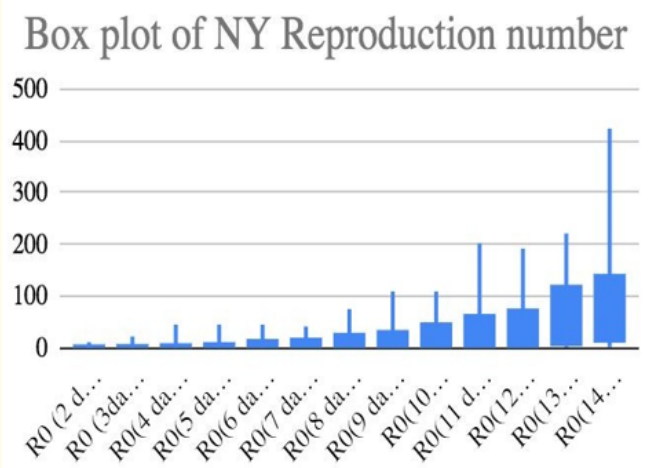

Figure 3

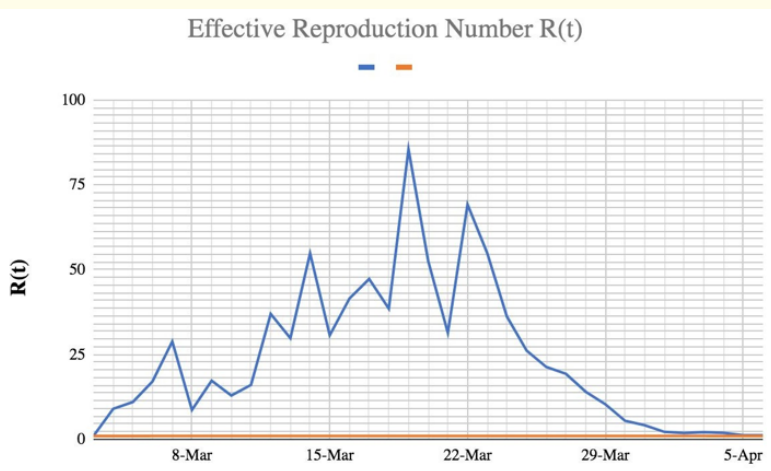

Figure 4

Infectivity parameter estimation: We used Latin Hypercube Simulation Partial Rank Correlation coefficient (LHS PRCC) to estimate infectivity parameters from both route symptotic Infectious and quarantine infectious NY populations. We estimated that infectivity rate from symptotic Infectious population is $\beta \mathrm{i} 1.5808(95 \%$ CI 1.4398 - 1.7218), and infectivity rate from Quarantine population is $\beta \mathrm{q}$ is 1.5754 (95\% $1.4336-1.7172)$ as well as death rate from Infectious class (symptotic and asymptotic - quarantine or not showing symptoms) is $\delta \mathrm{i}=0.00918$ (95\% CI $0.0091-0.00925$ ). To best fit the model with data from We found the $40.5 \%$ confirmed infected with incubation period to fit the model is 14 days for the NY population. The recovery parameter $\alpha$ at hospitalization treatment was observed at $9.9 \%$ with 45 days recovery time, and recovery parameter at home treatment was $14.12 \%$ with 18 days recovery time. Onset of symptoms to death from hospitalization is $2.5 \%$ in 30 days (best fit to model), and death from treatment at home is $0.92 \%$ in 14 days. All parameters values are mentioned in table 1 .

In this section, using Runge-Kutta-Fehlberg method of order 4 and 5, we numerically simulate our system (1) and (2) with real parametric values as given in table 1 and MATLAB is used to simulate the systems. We run the simulation for previous 55 days in consideration to fit the model, and it clearly indicates that major exposure occurs from the middle of January as we have data from March 1, 2020. We found the peak occurred on April 14, with 213,779 total confirmed cases. The trajectory of the graph in figure 6 predicted May 2, with a total 390,330 cumulative number of infectious population in New York, will be a crucial time when the graph has a sudden fall that indicates either faster recovery or death or both. Figure 7 validate the model with the current number of total infectious population, and number of deceased. The simulation predicted that by May 2, 2020, the number of the recovered population would be 205,800 , and deaths due to COVID-19 would be 60,860 as shown in figure 8 . Even our model is showing more recovered population which we believe is due to undocumented recovered population treated at home. The longevity of nature of infections predicted by Oct. 26, 2020 the probable number of cases will be approx. 4000, even for longer run of simulation it predicted the infection will be occur in control mode, that clearly indicate the forthcoming vaccine would be able to mitigate the severity of COVID-19 in the NY population.

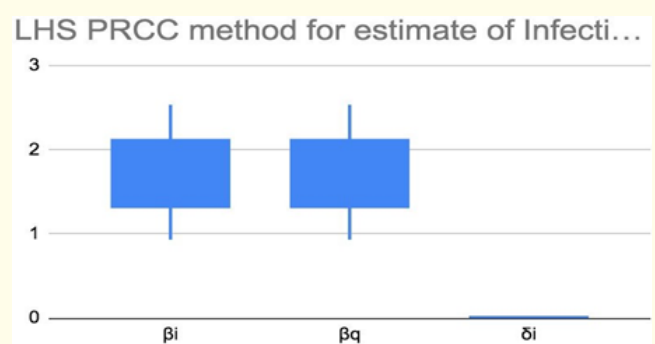

Figure 5 


\begin{tabular}{|l|l|l|}
\hline \multicolumn{1}{|c|}{ Parameters } & \multicolumn{1}{|c|}{ Value } & \multicolumn{1}{c|}{ Source } \\
\hline $\mathrm{B}$ & 0.00003397260274 & $(5)$ \\
\hline$\mu$ & 0.00002328767123 & $(6)$ \\
\hline$\eta$ & 0.02893645 & Calculated \\
\hline$\xi \mathrm{q}$ & 0.85879 & Calculated \\
\hline$\xi \mathrm{r}$ & 0.00784370624 & Calculated \\
\hline & $0.00918(95 \% \mathrm{CI}$ & \\
$\delta \mathrm{i}$ & $0.0091-0.00925)$ & Fitted \\
\hline$\delta \mathrm{q}$ & 0.024497405 & Calculated \\
\hline $\mathrm{a}$ & 0.09909932 & Calculated \\
\hline$\varepsilon$ & 0.0001111111111 & Assumed \\
\hline$\beta \mathrm{i}$ & $1.5808(95 \% \mathrm{CI} 1.4398-1.7218)$ & Fitted \\
\hline$\beta \mathrm{q}$ & $1.5754(95 \% 1.4336-1.7172)$ & Fitted \\
\hline $\mathrm{N}$ & 19491339 & 7 \\
\hline
\end{tabular}

Table 1: Parameters values and their source.

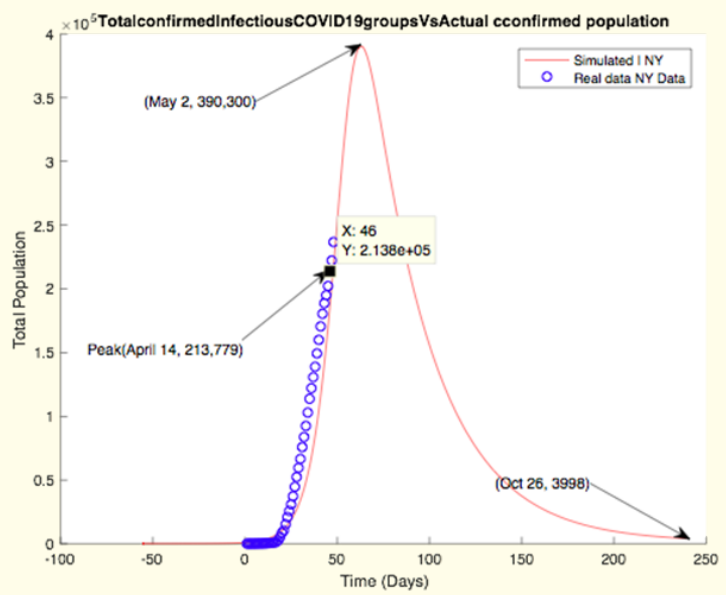

Figure 6

\section{Conclusion}

In this study, by applying the SEIQRD compartmental model to the daily reported cases of COVID-19 in New York from Mar. 1, 2020 to April 6, 2020, we have estimated that the basic reproduction number $\mathrm{R}_{0}=27.615695 \%$ CI [9.65301, 45.5781], while the median reproduction number ranges from 1.45 to 45 .We estimated a decline in $\mathrm{R}(\mathrm{t})$ from late March, from 41.483 (95\% CI 23.07 115.32) on March 22, the week the lockdown started, to 1.17 (95\%

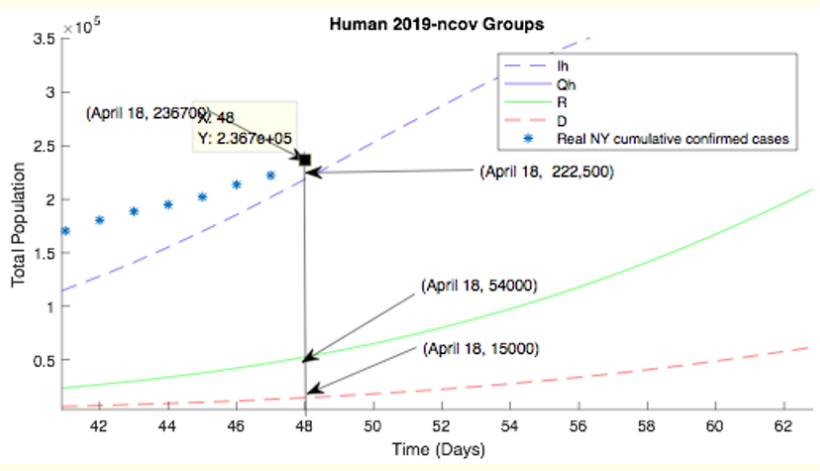

Figure 7

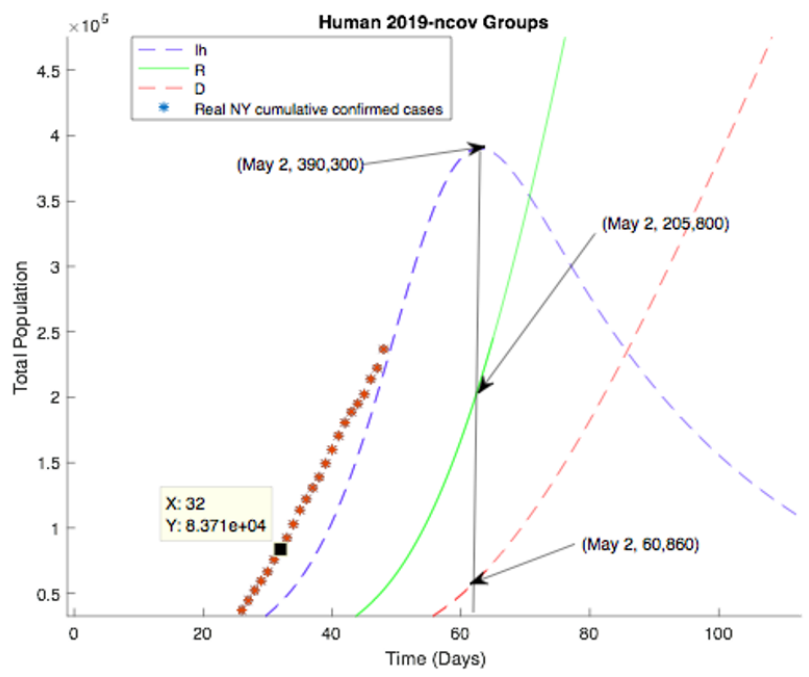

Figure 8

CI 1.05 - 1.38) on April 6, 2020. Our simulation has shown that the New York population have been exposed to COVID-19 from about January 14, 2020 that outcomes the large outbreak (without control) and have peak reproduction number 85 on Mar. 19, 2020, and once under control measure it could go down to 1.17 (95\% CI 1.05 - 1.38) on April 6, 2020. The model prediction is by May 2, 2020, the infectious population will be 390,300 , the recovered population 205,800 , and deaths due to COVID-19 would be 60,860, after a sudden drop in graphs which predicts both more recovery and more death in the infectious group. The projection of deaths is higher than reported deaths due to three possible reasons: (1) the clinical treat- 
ment level developed rapidly; (2) the demographics of the infected population changed, i.e. at the beginning of the pandemic, many of the most vulnerable people, such as those in nursing homes and people with pre-existing conditions, died; and (3) the reported data was not completely accurate due to many people dying with incorrect cause of death or those dying at home with no report. Its longevity suggests persistence of COVID-19 for an unpredicted time, while severity will go down but still a small number of cases would be occurring for a long time. So to prevent and control a great prevalence of COVID-19 and to minimize community spread, all precautions must be considered [1-11].

\section{Competing Interest}

The authors declare no competing interest.

\section{Author's Contribution}

Designed the project (D. S.); performed simulations (D. S.), performed analysis of the data (D. S., P. T..), wrote the paper (D. S., P. T.).

\section{Bibliography}

1. AB Gumel., et al. "Modelling strategies for controlling SARS outbreaks". Proceedings of the Royal Society 271.1554 (2004): 2223-2232.

2. G Chowell., et al. "Synthesizing data and models for the spread of MERS-CoV, 2013: key role of index cases and hospital transmission". Epidemics 9 (2014): 40-51.

3. ZQ Xia., et al. "Modeling the transmission of Middle East Respirator Syndrome Corona Virus in the Republic of Korea". PLoS ONE 10.12 (2015): 1-13.

4. Durgesh Sinha and Ankur Sinha. "Mathematical Model of Zoonotic NIPAH virus in South-East Asia Region". Acta Scientific Microbiology 2.9 (2019): 82-89.

5. https://www.indexmundi.com/g/g.aspx?c=us\&v=25

6. https://www.indexmundi.com/g/g.aspx?v=26\&c=us\&l=en

7. https://www.worldometers.info/world-population/us-population/

8. Covid19tracker.health.ny.gov
9. https://coronavirus.health.ny.gov/past-coronavirus-briefings

10. https://en.wikipedia.org/wiki/2020_coronavirus_pandemic_ in_New_York_(state)

11. Sinha Durgesh and Tan Peiwen. Mathematical Model and Simulations of COVID-19 2020 Outbreak in New York: Predictions and Implications for Control Measures (2020).

\section{Assets from publication with us}

- Prompt Acknowledgement after receiving the article

- Thorough Double blinded peer review

- Rapid Publication

- Issue of Publication Certificate

- High visibility of your Published work

Website: www.actascientific.com/

Submit Article: www.actascientific.com/submission.php

Email us: editor@actascientific.com

Contact us: +919182824667 